\author{
I. Camarda, G. Brundu, L. Carta, G. Vacca \& A. Brunu
}

\title{
Plants and grazing: an evaluation of the effects on Sardinian endemic plants conservation
}

\begin{abstract}
Camarda, I., Brundu, G., Carta, L., Vacca, G. \& Brunu, A.: Plants and grazing: an evaluation of the effects on Sardinian endemic plants conservation. - Fl. Medit. 25 (Special Issue): 263-275. 2015. - ISSN: 1120-4052 printed, 2240-4538 online.

We analysed the relationship between the 302 endemic entities of the Sardinian flora and the free-ranging domestic herbivores. This study highlights that the presence of spines, toxic compounds, resins, aromas and the adaptation to specific habitats are very effective defense traits that concur to the conservation of these species. The presence and abundance of $45.4 \%$ endemic entities of the Sardinian flora is not affected by grazing and browsing, while domestic herbivores promote the presence and abundance of $38.4 \%$ of endemic entities by reducing other competing species such as pabular herbs and woody species. However, grazing and browsing may threaten $16.2 \%$ of the endemic species. This study remarks the importance of the management of free-ranging domestic herbivores for effective conservation politics aiming to protect the endemic entities of the Sardinian flora.
\end{abstract}

Key words: life forms, traits, defense, domestic herbivores, Sardinia.

\section{Introduction}

The endemic species characterise the biological history of a given region. A comprehensive understanding of the vegetation dynamic successions and of the relationships between animal and plant is the basis for an effective policy for nature conservation. That relationship could be positive and synergic, as in the case of plant-pollinators, or negative, or controversial, as in the case of herbivores and endemic plants.

The Mediterranean ecosystems, as most ecosystems at global level, have been disturbed for at least three millennia by the presence of free-ranging domestic animals (Naveh 1974; Tomaselli 1976; Di Castri 1981; Camarda 1984; Dell \& al. 1986; Camarda 1991, 1992).

Domestic herbivores, in particular, have a specific impact on natural and semi-natural ecosystems in relation to the rangeland management system (Le Houérou 1981). Animal husbandry in Sardinia has affected most of the land in several ways (Le Lannou 1941) that have significantly shaped the vegetation. In Sardinia, the remains of domestic herbivores 
(sheep, mouflon, goat, cattle) are documented since the Neolithic period in several caves and are becoming more abundant in the Bronze Age until our time (Wilkens 2012). Their introduction could have caused the extinction of some botanic species, although this should be verified by data of paleobotanical research. In Sardinia, studies on relationship between plants and domestic animals has been only partially investigated (Aru \& al.1982; Camarda 1984, 1987, 1989, 1990; Farris \& Filigheddu 2008; Farris \& al. 2009; Pisanu \& al. 2009; Farris \& al. 2010; Pisanu \& al. 2012) despite its importance to different ecosystems. In the present study we focus on the endemic species of Sardinia. The availability of specific studies for this plant group (Arrigoni \& al. 1977-91; Arrigoni 2006-15) gives the possibility to estimate the impact of domestic herbivores in relation to the different farming systems and rangeland management (Pulina \& al. 2011; Pulina \& Biddau 2015), aiming to assess the actual influence of domestic herbivores on the Sardinian landscape (Ronchi \& al. 2013) and on the conservation of plant resources.

\section{Material and Methods}

In the last few decades, some areas formerly characterised by the free-ranging of domestic herbivores has been converted to more modern farming systems with sheds and other facilities that include the availability of extra-farm feeds. Nevertheless, the free-ranging of domestic herbivores is still a very common farming system, and grazing and browsing animals (cows, goats, sheep, pigs, horses, donkeys) use natural plant resources available in the rangelands or locally at the farm. In the present study, we did not take into account the presence and the effects of wild fauna, such as wild boars, mouflons (widespread in much of Sardinia), deers (a wild population is present in SW Sardinia), and roe deers (introduced in many areas of the island), foxes, pine marten and other small mammals such as oak rats.

Plant defensive traits are represented by functional adaptations to the local environment and by specific defensive traits against herbivores. In many cases, the same trait can be both an environmental adaptation and a defensive trait. The habitat type, such as rock outcrops and cliffs may be completely inaccessible to domestic herbivores, thus constituting a shelter that can protect the full plant life cycle. Geophytism is a life form that represents a natural adaptation to fire but also an effective defense against grazing and browsing. Geophytes with bulbs in the deep soil layers can survive for some time also in the absence of seed production. The presence of rhizomes, the prostrate or trailing habit, the presence of spines or hairiness, the production of toxic compounds (alkaloids, latex) or repellents (resins, aromas and flavours) are all very important defensive traits. The production of a high number of seeds is also an important indirect defense mechanism. Several defense traits can be shared by a single species to produce an effective defensive syndrome that could provide a competitive advantage in rangelands.

We took into account the entities that have a range restricted to Sardinia (endemic in the narrow sense) and also those species whose range includes Corsica, Balearic Islands, Tuscany Archipelago and Sicily. In a limited number of cases, we considered also those species which might be present also in the Italian southern regions facing 
the Tyrrhenian see. We did not consider those species with a range that includes Africa and continental Europe.

Mostly all plant species, whenever available forage resources are scarce, could be browsed by domestic herbivores. All the endemic entities of Fabaceae, Brassicaceae and Poaceae are edible and are often browsed, despite their specific differences. Different parts of the same species might be browsed differently. Plant species with toxic or irritant latex are usually avoided or browsed only occasionally. Other endemic species have alkaloids or glycosides that might be toxic (Helleborus argutifolius), irritant (Urtica atrovirens) or repellent, as in the case of Lamiaceae (despite having an agreeable smell to men). Many other species, belonging to different families, have a very small size and therefore are neglected by herbivores. As already mentioned, geophytism with the presence of bulbs or rhizomes buried in the soil is a defensive trait against grazing and browsing. Prostrate and sub-fruticose (sub-shrubs) growth forms are tolerant to browsing as well, especially when spines or thorns are present. Endemic and rare species, or species with a punctiform range, are of course the most threatened by grazing and browsing.

\section{Results}

Table 1 lists 302 entities (species, subspecies, varieties) and their main traits such as life forms and growth forms, their distribution in Sardinia, pabularity (yes/no), defensive traits against browsing. In addition, for each entity, we estimated whether their presence and abundance is promoted or reduced by browsing and grazing (see Tab. 1 and the respective graphics in Fig. 1 and Fig. 2).

\section{Life forms and growth forms}

The biological form of plant species expresses the conditions of adaptation to the environmental conditions as an effective response during the adverse season to its survival and development of the aerial parts of each species. This is also an effective defense to grazing (Camarda 1989). Hemicriptophytes (38.4\%), are the most common life form among the 302 endemic entities and present a high number of buds protected in different ways at the ground level. This mechanism favors the plant survival over time, and could be enhanced by the plant's shape, as in the case of caespitose (43.7\% over the total) whose basal part remains are a further protection for the basal overwintering buds. Chamaephytes (36.1\%) are the second most common life form. Pulviniform (cushion-shaped) chamaephytes have often thorny branches (Genista morisii, Berberis aetnensis) or thorny leaves (Astragalus terraccianoi, Centaurea horrida) acting as a defensive barrier protecting the full life cycle. Pulviniform chamaephytes can be a shelter for many other species (Polygala sinisica, Saponaria alsinoides, Lamium corsicum, Viola limbarae). Hemicrtyptophytes / Chamaephytes such as many species of Limonium, have often only a reduced leaf system and very small woody branches. The defense system may be further enhanced by specie leaf properties, branches or root system defensive traits. Endemic geophytes with bulbs of rhizomes buried and protected by the soils are the $13.2 \%$ of the total endemic species (e.g. Colchicum gonarei, Crocus minimus, 
Aristolochia tyrrhena). Therophytes are in general more exposed to herbivores, but for many of them the very small plant size (Herniaria litardierii, Euphrasia genargentea, Veronica brevistyla) or the very early cycle (before the presence of free-ranging animals in the mountain rangelands) or short life span are traits that allow them to be neglected by herbivores.

\section{Habitat}

The habitat type is a very important element to consider in relation to the conservation of the endemic species of the Sardinian flora. Some species are strictly casmophilous (Ptychotis sardoa, Campanula forsythii, Cymbalaria muelleri, Limonium morisianum, Saxifraga cervicornis), while other (Brassica insularis, Centaurea filiformis, Psoralea morisiana, Barbarea rupicola, Lactuca longidentata, Seseli praecox) are usually casmophilous but, if not disturbed by grazers, can thrive also at the bottom of rocks and cliffs.

\section{Thorns}

The presence of spines can only partly reduce the browsing of the species in the genus Genista, Anthyllis, Astragalus, while the seeds in the legumes of these species are very rich in starch and proteins. On the contrary, the large amount of spines on leaves, stems and inflorescences of Ptilostemon casabonae and Dipsacus valsecchiae constitutes the ultimate defense against browsing.

\section{Toxicity}

Some endemic species have high contents of alkaloids and glycosides (Aquilegia sp.pl., Paeonia morisii, Arum pictum) and, despite their rich shoot system, are completely avoided by domestic herbivores during the summer season. Helleborus argutifolius is always unpalatable, even when dry, due to the presence of spines on the leaves, and for the high content of alkaloids and glycosides that are toxic. Ruta corsica produces furocumarins that are very irritating (Ena \& Camarda 1990). Other species (Nepeta foliosa, Santolina insularis, Santolina corsica, Stachys glutinosa) produce aromas that may be agreeable to men but that are often repellent for domestic herbivores. Thymus catharinae is a species very rich in essential oils and as a result it is browsed only during the late summer period when there is less availability of other more palatable species. In spite of the very dense shoot systems, Vinca sardoa is always neglected by herbivores having a high content of toxic alkaloids.

\section{Rarity}

Browsing and grazing can seriously threaten rare and very rare endemic species, and those species with a punctiform range (Ribes sardoum, Rubus limbarae). This could increase extinction risk and surely reduce population size. Browsing and grazing should always be taken into account in the assessment of the conservation status of endangered species. 


\section{Conclusions}

The free range of domestic herbivores reduces the presence and abundance of woody and herbaceous edible species. At the same time this phenomen promotes the abundance of those endemic species which have defensive traits against browsing and grazing. This mechanism explains the local abundance of species such as Paeonia morisii, Astragalus genargenteus, Aquilegia sp.pl., Helleborus argutifolius, and Ruta corsica in areas strongly degraded by grazing, while being considered as rare species at Island level. Species lacking of defensive traits against browsing and grazing are in total $92(30.5 \%)$, mostly Poaceae (Festuca morisiana, Festuca sardoa, Sesleria insularis) but also belonging to other families (Scorzonera callosa, Hypochoeris robertia, Astragalus maritimus, Astragalus verrucosus, Borago pygmaea, Borago morisiana, Echium anchusoides). We list $156(51.7 \%)$ endemic species that can be browsed by domestic herbivores and 146 $(48.3 \%)$ species that do not elicit pabular interest or are completely avoided because of their very limited plant size or inaccessibility of the habitat. The presence and abundance of $137(45.4 \%)$ species is not affected by browsing and grazing, $116(38.4 \%)$ species are promoted by grazing; and only $49(16.2 \%)$ decrease because of the presence of domestic herbivores. Nevertheless, these relationships are influenced by the species of domestic herbivores, the number of grazing animals, the presence of wild fauna, which could favor the spread of some seeds or propagules. Other driving forces are the anthropic activities, the road network, the movement of soil or other substrata that could promote the local presence of species such as Tanacetum audiberti, Dipsacus valsecchiae, Hieracium templare, Ptilostemon casabonae. Urtica atrovirens thrives very well close to sheep farms, specifically in sites where there is a high content of organic matter.

The present study highlights the complexity of the exciting relationships between freeranging domestic herbivores and the endemic entities of the Sardinian flora. The knowledge of these relationships is a fundamental basis for conservation politics and action aiming to protect endemic species in rangelands both in Sardinia and in the Mediterranean region.

Table 1. The table shows endemic species with their life forms and growth forms and attributes regards relationships between pabularity, defense types, kind of presence (com. $=$ common; freq. $=$ frequent; spor.=sporadic; punct. $=$ punctiform; rare/ab.= rare but locally abundant; v.r. $=$ very rare) and favorite by grazing ( fav = favorite; unfav = unfavorite; ind $=$ indifferent $)$.

\begin{tabular}{|c|c|c|c|c|c|c|c|c|}
\hline No. & Family & Taxon & 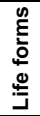 & 돈 & $\begin{array}{l}\frac{3}{2} \\
\frac{\pi}{5} \\
\frac{0}{\pi} \\
0\end{array}$ & 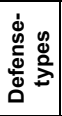 & 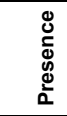 & 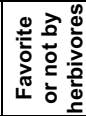 \\
\hline 1 & Lamiaceae & Acinos sardous (Asch. \& Levier) Arrigoni & $\overline{\mathrm{H}}$ & scap & no & arom & rare & fav \\
\hline 2 & Liliaceae & Allium parciflorum Viv. & G & bulb & no & arom & com. & fav \\
\hline 3 & Liliaceae & Allium roseum var. insulare Gennari & G & bulb & no & arom & com. & fav \\
\hline 4 & Brassicaceae & Alyssum tavolarae Briquet & $\mathrm{Ch}$ & caesp & no & hab & rare & ind \\
\hline 5 & Boraginaceae & Anchusa capellii Moris & $\mathrm{H}$ & caesp & yes & no & rare & ind \\
\hline 6 & Boraginaceae & Anchusa crispa Viv. & $\mathrm{H}$ & caesp & no & no & rare & ind \\
\hline 7 & Boraginaceae & Anchusa maritima Valsecchi & $\mathrm{T}$ & caesp & no & no & rare & ind \\
\hline 8 & Boraginaceae & Anchusa formosa Selvi, Bigazzi \& Bacchetta & $\mathrm{H}$ & caesp & yes & no & punct. & unfav \\
\hline 9 & Boraginaceae & Anchusa littorea Moris & $\mathrm{H}$ & caesp & no & no & rare & unfav \\
\hline 10 & Boraginaceae & Anchusa montelinasana Angius, Pontecorvo \& Selvi & $\mathrm{H}$ & caesp & yes & no & rare & unfav \\
\hline 11 & Boraginaceae & Anchusa sardoa (Illario) Selvi \& Bigazzi & $\mathrm{H}$ & prost & yes & no & v.r. & ind \\
\hline 12 & Fabaceae & Anthyllis hermanniae subsp. ichnusae Brullo \& Giusso & $\mathrm{Ch}$ & pulv & no & spin & rare & ind \\
\hline 13 & Ranunculaceae & Aquilegia barbaricina Arrigoni \& Nardi & $\mathrm{H}$ & scap & no & tox & punct. & ind \\
\hline 14 & Ranunculaceae & Aquilegia nugorensis Arrigoni \& Nardi & $\mathrm{H}$ & scap & no & tox & punct. & ind \\
\hline
\end{tabular}


Table 1. continued.

\begin{tabular}{|c|c|c|c|c|c|c|c|c|}
\hline No. & Family & Taxon & $\begin{array}{l}\stackrel{n}{E} \\
\stackrel{5}{0} \\
\stackrel{0}{\longleftarrow}\end{array}$ & 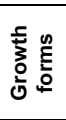 & $\begin{array}{l}\frac{2}{2} \\
\frac{\pi}{\pi} \\
\frac{0}{\pi} \\
0 \\
0\end{array}$ & 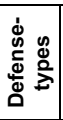 & 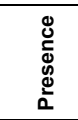 & 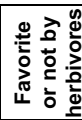 \\
\hline 15 & Ranunculaceae & Aquilegia nuragica Arrigoni \& Nardi & $\mathrm{H}$ & scap & no & tox & punct. & ind \\
\hline 16 & Caryophyllaceae & Arenaria balearica $\mathrm{L}$ & $\mathrm{H}$ & rept & no & hab & freq. & ind \\
\hline 17 & Aristolochiaceae & Aristolochia tyrrhena Nardi \& Arrigoni & G & rhiz & no & tox & rare & fav \\
\hline 18 & Plumbaginaceae & Armeria morisii Boiss. in A. DC. & $\mathrm{H}$ & caesp & yes & hab & rare & fav \\
\hline 19 & Plumbaginaceae & Armeria sardoa subsp. genargentea Arrigoni & $\mathrm{H}$ & caesp & yes & hab & v.r. & fav \\
\hline 20 & Plumbaginaceae & Armeria sardoa Sprengel subsp. sardoa & $\mathrm{H}$ & caesp & yes & hab & rare & fav \\
\hline 21 & Plumbaginaceae & Armeria sulcitana Arrigoni & $\mathrm{H}$ & caesp & yes & hab & v.r. & fav \\
\hline 22 & Asteraceae & Artemisia variabilis Ten. & $\mathrm{Ch}$ & caesp & no & arom & rare & fav \\
\hline 23 & Asteraceae & Artemisia corensis Vals. \& Filigh. & $\mathrm{Ch}$ & caesp & no & arom & rare & fav \\
\hline 24 & Asteraceae & Artemisia densiflora Viv. & $\mathrm{Ch}$ & caesp & no & arom & v.r. & fav \\
\hline 25 & Araceae & Arum pictum L. fil. & G & rhiz & no & tox & com. & fav \\
\hline 26 & Rubiaceae & Asperula deficiens Viv. & $\mathrm{H}$ & rept & no & hab & punct. & ind \\
\hline 27 & Rubiaceae & Asperula pumila Moris & $\mathrm{H}$ & caesp & no & hab & v.r. & ind \\
\hline 28 & Fabaceae & Astragalus genargenteus Moris subsp. genargenteus & $\mathrm{Ch}$ & pulv & yes & spin & rare/ab. & fav \\
\hline 29 & Fabaceae & Astragalus genargenteus subsp. gennarii (Bacchetta \& Brullo) Arrigoni & $\mathrm{Ch}$ & pulv & yes & spin & rare & fav \\
\hline 30 & Fabaceae & Astragalus rnaritimus Moris & $\mathrm{H}$ & scap & yes & spin & punct. & fav \\
\hline 31 & Fabaceae & Astragalus terraccianoi Valsecchi & $\mathrm{Ch}$ & pulv & yes & spin & rare & fav \\
\hline 32 & Fabaceae & Astragalus thermensis Valsecchi & $\mathrm{Ch}$ & pulv & yes & spin & punct. & fav \\
\hline 33 & Fabaceae & Astragalus verrucosus Moris & $\mathrm{T}$ & scap & yes & no & rare & unfav \\
\hline 34 & Brassicaceae & Barbarea rupicola Moris & $\mathrm{H}$ & scap & yes & hab & spor. & unfav \\
\hline 35 & Asteraceae & Bellium bellidioides L. & $\mathrm{H}$ & rept & yes & hab & com. & ind \\
\hline 36 & Asteraceae & Bellium crassifolium Moris & $\mathrm{H}$ & ros & yes & hab & rare & unfav \\
\hline 37 & Berberidaceae & Berberis aetnensis C. B. Presl & $\mathrm{Ch}$ & caesp & no & spin & rare & fav \\
\hline 38 & Brassiceae & Biscutella morisiana Raffaelli & $\mathrm{T}$ & scap & yes & no & rare & unfav \\
\hline 39 & Boraginaceae & Borago morisiana Bigazzi \& Ricceri & $\mathrm{H}$ & scap & yes & no & spor. & unfav \\
\hline 40 & Boraginaceae & Borago pygmaea (DC.) Chater \& Greuter & $\mathrm{H}$ & scap & yes & no & spor. & unfav \\
\hline 41 & Brassicaceae & Brassica insularis Moris & $\mathrm{Ch}$ & suff & yes & hab & rare & unfav \\
\hline 42 & Brassicaceae & Brassica thyrrhena Giotta, Piccitto \& Arrigoni & $\mathrm{Ch}$ & suff & yes & hab & com. & unfav \\
\hline 43 & Cucurbitacea & Bryonia marmorata Petit & G & lian & no & hab & com. & unfav \\
\hline 44 & Boraginaceae & Buglossoides minima (Moris) R. Fernandes & $\mathrm{T}$ & scap & yes & no & rare & fav \\
\hline 45 & Asteraceae & Buphthalmum inuloides Moris & $\mathrm{Ch}$ & caesp & yes & arom & rare & fav \\
\hline 46 & Amiryllidaceae & Narcissus supramontanus Arrigoni & G & bulb & no & tox & rare/ab. & fav \\
\hline 47 & Amiryllidaceae & Narcissus supramontanus subsp. cunicalarius & G & bulb & no & tox & rare & fav \\
\hline 48 & Lamiaceae & Calamintha sandaliotica Bacchetta \& Brullo & $\mathrm{H}$ & scap & yes & arom & spor. & ind \\
\hline 49 & Campanulaceae & Campanula forsythii (Arcangeli) Podlech & $\mathrm{H}$ & caesp & yes & hab & rare & ind \\
\hline 50 & Asteraceae & Carduus corymbosus Ten. & $\mathrm{H}$ & scap & yes & spin & com. & fav \\
\hline 51 & Asteraceae & Carduus fasciculiflorus Viv. & $\mathrm{H}$ & scap & yes & spin & rare & fav \\
\hline 52 & Asteraceae & Carduus sardous DC. & $\mathrm{T}$ & scap & yes & spin & rare & fav \\
\hline 53 & Cyperaceae & Carex caryophyllea subsp. insularis (Christ ex Barbey) Arrigoni & $\mathrm{H}$ & caesp & yes & no & rare & unfav \\
\hline 54 & Cyperaceae & Carex microcarpa Bertol. ex Moris & $\mathrm{H}$ & caesp & yes & spin & rare & fav \\
\hline 55 & Cyperaceae & Carex panormitana Guss. & $\mathrm{H}$ & scap & no & spin & v.r. & fav \\
\hline 56 & Asteraceae & Carlina macrocephala Moris & $\mathrm{Ch}$ & scap & yes & spin & punct. & fav \\
\hline 57 & Asteraceae & Centaurea corensis Filigheddu \& Valsecchi & $\mathrm{Ch}$ & suff & yes & arom & rare & fav \\
\hline 58 & Asteraceae & Centaurea filiformis subsp. ferulacea (Martelli) Arrigoni & $\mathrm{Ch}$ & suff & yes & hab & rare & ind \\
\hline 59 & Asteraceae & Centaurea filiformis Viv. subsp. filiformis & $\mathrm{Ch}$ & caesp & yes & hab & rare & ind \\
\hline 60 & Asteraceae & Centaurea horrida Bad. & $\mathrm{Ch}$ & pulv & yes & spin & rare/ab. & unfav \\
\hline 61 & Asteraceae & Centaurea magistrorum Arrigoni \& Camarda & $\mathrm{Ch}$ & pulv & no & arom & v.r. & fav \\
\hline 62 & Valerianaceae & Centranthus amazonum Friedlender & $\mathrm{H}$ & scap & yes & hab & punct. & unfav \\
\hline 63 & Dipsacaceae & Cephalaria bigazzii Bacchetta, Brullo \& Giusso del Galdo & $\mathrm{Ch}$ & scap & yes & hab & rare & ind \\
\hline 64 & Dipsacaceae & Cephalaria mediterranea (Viv.) Szabò & $\mathrm{Ch}$ & scap & no & hab & rare & ind \\
\hline 65 & Caryophyllaceae & Cerastium palustre Moris & $\mathrm{T}$ & scap & yes & no & rare & fav \\
\hline 66 & Caryophyllaceae & Cerastium supramontanum Arrigoni & $\mathrm{H}$ & caesp & yes & hab & rare & fav \\
\hline 67 & Colchicaceae & Colchicum actupii Fridl. & G & bulb & yes & tox & rare & fav \\
\hline 68 & Colchicaceae & Colchicum gonarei Camarda & G & bulb & yes & tox & rare & fav \\
\hline 69 & Colchicaceae & Colchicum verlaqueae Fridl. & G & bulb & yes & tox & rare & fav \\
\hline 70 & Iridaceae & Crocus minimus DC. in Rédouté & G & bulb & no & hab & com. & fav \\
\hline 71 & Scrophulariaceae & Cymbalaria aequitriloba (Viv.) A. Chevalier & $\mathrm{H}$ & rept & yes & hab & rare & ind \\
\hline 72 & Scrophulariaceae & Cymbalaria muelleri (Moris) A. Chevalier & $\mathrm{H}$ & caesp & no & hab & v.r. & ind \\
\hline 73 & Ranunculaceae & Delphinium longipes Moris & $\mathrm{H}$ & scap & no & tox & rare & fav \\
\hline 74 & Ranunculaceae & Delphinium pictum Willd. & $\mathrm{H}$ & scap & no & tox & rare & fav \\
\hline 75 & Caryophyllaceae & Dianthus siculus subsp. cyatophorus (Moris) Arrigoni & $\mathrm{Ch}$ & caesp & yes & hab & rare & fav \\
\hline 76 & Caryophyllaceae & Dianthus siculus subsp. morisianus (Valsecchi) Arrigoni & $\mathrm{Ch}$ & caesp & yes & no & rare & fav \\
\hline 77 & Caryophyllaceae & Dianthus siculus subsp. mossanus (Bacchetta \& Brullo) Arrigoni & $\mathrm{Ch}$ & caesp & yes & hab & rare & fav \\
\hline 78 & Caryophyllaceae & Dianthus siculus subsp. stellaris (Camarda) Arrigoni & $\mathrm{Ch}$ & caesp & yes & hab & rare & fav \\
\hline 79 & Caryophyllaceae & Dianthus siculus subsp. tenuifolius (Moris) Arrigoni & $\mathrm{Ch}$ & caesp & no & hab & rare & fav \\
\hline 80 & Dipsacaceae & Dipsacus ferox Loisel. & $\mathrm{H}$ & scap & no & spin & rare & fav \\
\hline 81 & Dipsacaceae & Dipsacus valsecchiae Camarda & $\mathrm{H}$ & scap & no & spin & rare & fav \\
\hline 82 & Araceae & Dracunculus muscivorus (L. fil.) Parlatore & G & scap & no & tox & rare & fav \\
\hline 83 & Boraginaceae & Echium anchusoides Bacchetta, Brullo \& Selvi & $\mathrm{H}$ & rept & yes & no & rare & unfav \\
\hline 84 & Geraniaceae & Erodium corsicum Léman in Lam. \& DC. & $\mathrm{H}$ & rept & yes & hab & com. & fav \\
\hline 85 & Geraniaceae & Erodium nervulosum L'Hér. & $\mathrm{H}$ & prost & yes & hab & rare & ind \\
\hline 86 & Euphorbiaceae & Euphorbia cupanii Guss. ex Bertol. & $\mathrm{T}$ & caesp & no & tox & punct. & fav \\
\hline 87 & Euphorbiaceae & Euphorbia insularis Boiss. & $\mathrm{H}$ & caesp & no & tox & rare & fav \\
\hline 88 & Euphorbiaceae & Euphorbia meuselii Mazzola \& Raimondo & $\mathrm{H}$ & caesp & no & tox & spor. & fav \\
\hline
\end{tabular}


Table 1. continued.

\begin{tabular}{|c|c|c|c|c|c|c|c|c|}
\hline No. & Family & Taxon & 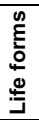 & 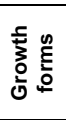 & $\begin{array}{l}\text { 를 } \\
\frac{0}{5} \\
\frac{0}{\pi} \\
0 \\
0\end{array}$ & 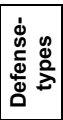 & 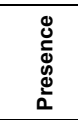 & 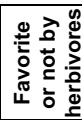 \\
\hline 89 & Euphorbiaceae & Euphorbia semiperfoliata Viv. & 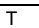 & caesp & no & tox & rare & fav \\
\hline 90 & Scrophulariaceae & Euphrasia genargentea (Feoli) Diana & $\mathrm{T}$ & scap & no & no & rare & fav \\
\hline 91 & Asteraceae & Evax rotundata Moris & $\mathrm{H}$ & scap & no & no & spor. & ind \\
\hline 92 & Apiaceae & Ferula arrigonii Bocchieri & $\mathrm{H}$ & scap & no & tox & rare & fav \\
\hline 93 & Poaceae & Festuca morisiana Parl. & $\mathrm{H}$ & caesp & yes & no & rare & fav \\
\hline 94 & Poaceae & Festuca sardoa (Hackel in Barbey) Richter & $\mathrm{H}$ & caesp & yes & no & rare/ab. & fav \\
\hline 95 & Rubiaceae & Galium corsicum Sprengel & $\mathrm{H}$ & prost & yes & hab & rare & fav \\
\hline 96 & Rubiaceae & Galium glaucophyllum E.Schmid & $\mathrm{H}$ & prost & yes & hab & rare & fav \\
\hline 97 & Rubiaceae & Galium schmidii Arrigoni & $\mathrm{T}$ & prost & no & hab & rare & fav \\
\hline 98 & Fabaceae & Genista aetnensis (Biv.) DC. & $\mathrm{P}$ & scap & yes & spin & com. & unfav \\
\hline 99 & Fabaceae & Genista arbusensis Valsecchi & $\mathrm{Ch}$ & caesp & no & spin & com. & fav \\
\hline 100 & Fabaceae & Genista bocchierii Bacchetta, Brullo \& Feoli Chiapella & $\mathrm{Ch}$ & caesp & no & spin & rare & fav \\
\hline 101 & Fabaceae & Genista cadasonensis Valsecchi & $\mathrm{Ch}$ & caesp & no & spin & com. & fav \\
\hline 102 & Fabaceae & Genista corsica (Loisel.) DC. in Lam. \& DC. & $\mathrm{Ch}$ & caesp & no & spin & com. & fav \\
\hline 103 & Fabaceae & Genista desoleana Valsecchi & $\mathrm{Ch}$ & caesp & no & spin & com. & fav \\
\hline 104 & Fabaceae & Genista ephedroides DC. & $\mathrm{P}$ & caesp & no & spin & rare & fav \\
\hline 105 & Fabaceae & Genista insularis Bacchetta, Brullo \& Feoli Chiapella & $\mathrm{Ch}$ & caesp & no & spin & rare & fav \\
\hline 106 & Fabaceae & Genista insularis subsp. fodinae Bacchetta, Brullo \& al. & $\mathrm{Ch}$ & caesp & no & spin & rare & fav \\
\hline 107 & Fabaceae & Genista ovina Bacchetta, Brullo \& Feoli Chiapella & $\mathrm{Ch}$ & caesp & no & spin & com. & fav \\
\hline 108 & Fabaceae & Genista morisii Colla & $\mathrm{Ch}$ & caesp & no & spin & com. & fav \\
\hline 109 & Fabaceae & Genista pichi-sermolliana Valsecchi & $\mathrm{P}$ & caesp & no & spin & com. & fav \\
\hline 110 & Fabaceae & Genista salzmannii DC. & $\mathrm{Ch}$ & caesp & no & spin & rare & fav \\
\hline 111 & Fabaceae & Genista sardoa Valsecchi & $\mathrm{Ch}$ & caesp & no & spin & rare & fav \\
\hline 112 & Fabaceae & Genista sulcitana Valsecchi & $\mathrm{Ch}$ & caesp & no & spin & rare & fav \\
\hline 113 & Fabaceae & Genista toluensis Valsecchi & $\mathrm{Ch}$ & caesp & no & spin & rare & fav \\
\hline 114 & Lamiaceae & Glechoma sardoa (Bég.) Bég. & $\mathrm{Ch}$ & caesp & yes & hab & rare & ind \\
\hline 115 & Cistaceae & Helianthemum allionii Tineo & $\mathrm{Ch}$ & caesp & yes & hab & rare & unfav \\
\hline 116 & Cistaceae & Helianthemum morisianum Bertol. & $\mathrm{Ch}$ & caesp & yes & hab & rare & unfav \\
\hline 117 & Asteraceae & Castroviejoa montelinasana E.Schmid & $\mathrm{Ch}$ & caesp & yes & hab & rare & ind \\
\hline 118 & Asteraceae & Helichrysum saxatile Moris & $\mathrm{Ch}$ & caesp & yes & hab & rare & ind \\
\hline 119 & Asteraceae & Helichrysum saxatile subsp. morisianum Bacchetta \& al. & $\mathrm{H}$ & caesp & no & hab & spor. & fav \\
\hline 120 & Ranunculaceae & Helleborus argutifolius Viv. & $\mathrm{H}$ & caesp & no & tox & rare & fav \\
\hline 121 & Caryophyllaceae & Herniaria litardierei (Gamis.) Greuter \& Burdet & $\mathrm{H}$ & scap & yes & no & rare & fav \\
\hline 122 & Asteraceae & Hieracium gallurense Arrigoni & $\mathrm{H}$ & scap & yes & no & rare & unfav \\
\hline 123 & Asteraceae & Hieracium iolai Arrigoni & $\mathrm{H}$ & scap & yes & no & rare & ind \\
\hline 124 & Asteraceae & Hieracium irginianum Arrigoni & $\mathrm{H}$ & scap & yes & no & rare & ind \\
\hline 125 & Asteraceae & Hieracium limbarae Arrigoni & $\mathrm{H}$ & scap & yes & no & rare & unfav \\
\hline 126 & Asteraceae & Hieracium martellianum (Zahn) Arrigoni & $\mathrm{H}$ & scap & yes & no & rare & ind \\
\hline 127 & Asteraceae & Hieracium oliastrae Arrigoni & $\mathrm{H}$ & scap & yes & no & rare & ind \\
\hline 128 & Asteraceae & Hieracium soleirolianum Arv. Touv. \& Briquet & $\mathrm{H}$ & scap & yes & no & rare & ind \\
\hline 129 & Asteraceae & Hieracium supramontanum Arrigoni & $\mathrm{H}$ & scap & yes & hab & rare & ind \\
\hline 130 & Asteraceae & Hieracium templare Arrigoni & $\mathrm{H}$ & scap & yes & no & com. & unfav \\
\hline 131 & Asteraceae & Hieracium zizianum subsp. sardonium Zahn & $\mathrm{H}$ & scap & yes & no & com. & unfav \\
\hline 132 & Hypericaceae & Hypericum annulatum Moris & $\mathrm{Ch}$ & caesp & no & tox & rare & fav \\
\hline 133 & Hypericaceae & Hypericum hircinum L. subsp. hircinum & $\mathrm{P}$ & caesp & yes & tox & com. & ind \\
\hline 134 & Asteraceae & Hypochaeris linearifolia Moris & $\mathrm{H}$ & caesp & yes & hab & rare & unfav \\
\hline 135 & Brassicaceae & Iberis integerrima Moris & $\mathrm{H}$ & prost & yes & no & rare & unfav \\
\hline 136 & Asteraceae & Lactuca longidentata Moris & $\mathrm{H}$ & scap & yes & hab & rare & unfav \\
\hline 137 & Lamiaceae & Lamium corsicum Godr. \& Gren. & $\mathrm{H}$ & caesp & yes & hab & rare & fav \\
\hline 138 & Asteraceae & Lamyropsis microcephala (Moris) Dittrich \& Greuter & $\mathrm{H}$ & caesp & yes & spin & v.r. & ind \\
\hline 139 & Malvaceae & Lavatera plazzae Atzei & $\mathrm{H}$ & caesp & yes & no & rare & unfav \\
\hline 140 & Malvaceae & Lavatera triloba subsp. pallescens (Moris) Nyman & $\mathrm{P}$ & scap & yes & no & rare & unfav \\
\hline 141 & Amaryllidaceae & Leucojum roseum Martin & G & bulb & no & no & rare/ab. & fav \\
\hline 142 & Plumbaginaceae & Limonium acutifolium subsp. cornusianum (Arrigoni \& Diana) Arrigoni & $\mathrm{Ch}$ & caesp & no & hab & rare & ind \\
\hline 143 & Plumbaginaceae & Limonium acutifolium subsp. obtusifolium (Rouy) Diana & $\mathrm{Ch}$ & caesp & no & hab & rare & ind \\
\hline 144 & Plumbaginaceae & Limonium acutifolium subsp. acutifolium & $\mathrm{Ch}$ & caesp & no & hab & rare & ind \\
\hline 145 & Plumbaginaceae & Limonium acutifolium subsp. bosanum (Arrigoni \& Diana) Arrigoni & $\mathrm{Ch}$ & caesp & no & hab & rare & ind \\
\hline 146 & Plumbaginaceae & Limonium acutifolium subsp. nymphaeum (Erben) Arrigoni & $\mathrm{Ch}$ & caesp & no & hab & rare & ind \\
\hline 147 & Plumbaginaceae & Limonium acutifolium subsp. tenuifolium (Bertol.ex Moris) Arrigoni & $\mathrm{Ch}$ & caesp & no & hab & rare & ind \\
\hline 148 & Plumbaginaceae & Limonium acutifolium subsp. tharrosianum (Arrigoni \& Diana) Arrigoni & $\mathrm{Ch}$ & caesp & no & hab & rare & ind \\
\hline 149 & Plumbaginaceae & Limonium ampuriense Arrigoni \& Diana & $\mathrm{Ch}$ & caesp & no & hab & rare & ind \\
\hline 150 & Plumbaginaceae & Limonium angustifolium (Tausch) Degen & $\mathrm{Ch}$ & caesp & no & hab & rare & ind \\
\hline 151 & Plumbaginaceae & Limonium articulatum (Loisel.) O.Kuntze & $\mathrm{Ch}$ & caesp & no & hab & punct. & ind \\
\hline 152 & Plumbaginaceae & Limonium capitis-eliae Erben & $\mathrm{Ch}$ & caesp & no & hab & rare & ind \\
\hline 153 & Plumbaginaceae & Limonium capitis-marci Arrigoni \& Diana & $\mathrm{Ch}$ & caesp & no & hab & punct. & ind \\
\hline 154 & Plumbaginaceae & Limonium carisae Erben & $\mathrm{Ch}$ & caesp & no & hab & rare & ind \\
\hline 155 & Plumbaginaceae & Limonium contortirameum (Mabille) Erben & $\mathrm{Ch}$ & caesp & no & hab & freq. & ind \\
\hline 156 & Plumbaginaceae & Limonium coralliforme Mayer & $\mathrm{Ch}$ & caesp & no & hab & rare & ind \\
\hline 157 & Plumbaginaceae & Limonium cornusianum Arrigonl \& Diana & $\mathrm{Ch}$ & caesp & no & hab & rare & ind \\
\hline 158 & Plumbaginaceae & Limonium cunicularium Arrigoni \& Diana & $\mathrm{Ch}$ & caesp & no & hab & rare & ind \\
\hline 159 & Plumbaginaceae & Limonium dictyocladum (Boiss. in A. DC.) O. Kuntze & $\mathrm{Ch}$ & caesp & no & hab & rare & ind \\
\hline 160 & Plumbaginaceae & Limonium dubium (Andr. ex Guss.) R. Lit. & $\mathrm{Ch}$ & caesp & no & hab & rare & ind \\
\hline 161 & Plumbaginaceae & Limonium gallurense Arrigoni \& Diana & $\mathrm{Ch}$ & caesp & no & hab & rare & ind \\
\hline 162 & Plumbaginaceae & Limonium glomeratum (Tausch) Erben & $\mathrm{Ch}$ & caesp & no & hab & freq. & ind \\
\hline
\end{tabular}


Table 1. continued.

\begin{tabular}{|c|c|c|c|c|c|c|c|c|}
\hline No. & Family & Taxon & $\begin{array}{l}\stackrel{n}{E} \\
\stackrel{5}{0} \\
\stackrel{0}{ \pm}\end{array}$ & 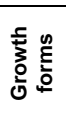 & $\begin{array}{l}\frac{7}{\frac{7}{\pi}} \\
\frac{\pi}{5} \\
\frac{0}{\pi} \\
0\end{array}$ & 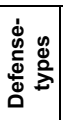 & 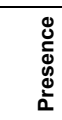 & 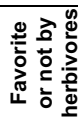 \\
\hline 163 & Plumbaginaceae & Limonium hermaeum (Pignatti) Pignatti & (Ch & caesp & no & hab & rare & ind \\
\hline 164 & Plumbaginaceae & Limonium insulare (Bég. \& Landi) Arrigoni \& Diana & $\mathrm{Ch}$ & caesp & no & hab & rare & ind \\
\hline 165 & Plumbaginaceae & Limonium laetum (Nyman) Pignatti & $\mathrm{Ch}$ & caesp & no & hab & v.r. & ind \\
\hline 166 & Plumbaginaceae & Limonium lausianum Pignatti & $\mathrm{Ch}$ & caesp & no & hab & punct. & ind \\
\hline 167 & Plumbaginaceae & Limonium malfatanicum Erben & $\mathrm{Ch}$ & caesp & no & hab & rare & ind \\
\hline 168 & Plumbaginaceae & Limonium merxmuelleri subsp. oristanum (Mayer) Arrigoni & $\mathrm{Ch}$ & caesp & no & hab & freq. & ind \\
\hline 169 & Plumbaginaceae & Limonium merxmuelleri subsp. sulcitanum (Arrigoni) Arrigoni & $\mathrm{Ch}$ & caesp & no & hab & rare & ind \\
\hline 170 & Plumbaginaceae & Limonium merxmuelleri subsp. tigulianum (Arrigoni \& Diana) Arrigoni & $\mathrm{Ch}$ & caesp & no & hab & rare & ind \\
\hline 171 & Plumbaginaceae & Limonium merxmuelleri Erben subsp. merxmuelleri & $\mathrm{Ch}$ & caesp & no & hab & spor. & ind \\
\hline 172 & Plumbaginaceae & Limonium morisianum Arrigoni & $\mathrm{Ch}$ & caesp & no & hab & punct. & ind \\
\hline 173 & Plumbaginaceae & Limonium multifurcatum Erben & $\mathrm{Ch}$ & caesp & no & hab & rare & ind \\
\hline 174 & Plumbaginaceae & Limonium protohermaeum Arrigoni \& Diana & $\mathrm{Ch}$ & caesp & no & hab & rare & ind \\
\hline 175 & Plumbaginaceae & Limonium pseudolaetum Arrigoni \& Diana & $\mathrm{Ch}$ & caesp & no & hab & rare & ind \\
\hline 176 & Plumbaginaceae & Limonium pulviniforme Arrigoni \& Diana & $\mathrm{Ch}$ & caesp & no & hab & rare & ind \\
\hline 177 & Plumbaginaceae & Limonium racemosum (Lojac.) Diana & $\mathrm{Ch}$ & caesp & no & hab & rare & ind \\
\hline 178 & Plumbaginaceae & Limonium retirameum susp. calaritanum (Erben) Arrigoni & $\mathrm{Ch}$ & caesp & no & hab & rare & ind \\
\hline 179 & Plumbaginaceae & Limonium retirameum Greuter \& Burdet subsp. retirameum & $\mathrm{Ch}$ & caesp & no & hab & rare & ind \\
\hline 180 & Plumbaginaceae & Limonium strictissimum (Salzmann) Arrigoni & $\mathrm{Ch}$ & caesp & no & hab & punct. & ind \\
\hline 181 & Plumbaginaceae & Limonium tibulatium Pignatti & $\mathrm{Ch}$ & caesp & no & hab & punct. & ind \\
\hline 182 & Plumbaginaceae & Limonium tigulianum Arrigoni \& Diana & $\mathrm{Ch}$ & caesp & no & hab & rare & ind \\
\hline 183 & Plumbaginaceae & Limonium tyrrhenicum Arrigoni \& Diana & $\mathrm{Ch}$ & caesp & no & hab & rare & ind \\
\hline 184 & Plumbaginaceae & Limonium ursanum Erben & $\mathrm{Ch}$ & caesp & no & hab & rare & ind \\
\hline 185 & Scrophulariaceae & Limonium viniolae Arrigoni \& Diana & $\mathrm{Ch}$ & caesp & no & hab & v.r. & ind \\
\hline 186 & Scrophulariaceae & Linaria arcusangeli Atzei \& Camarda & $\mathrm{Ch}$ & caesp & yes & hab & rare & unfav \\
\hline 187 & Scrophulariaceae & Linaria flava subsp. sardoa (Sommier) Arrigoni & $\mathrm{T}$ & scap & yes & hab & rare & ind \\
\hline 188 & Linaceae & Linum muelleri Moris & $\mathrm{T}$ & scap & yes & no & rare & ind \\
\hline 189 & Caprifoliaceae & Lonicera cyrenaica Viv. & $\mathrm{P}$ & lian & yes & hab & rare & unfav \\
\hline 190 & Cyperaceae & Luzula italica Parl. & $\mathrm{H}$ & scand & yes & no & rare & unfav \\
\hline 191 & Lamiaceae & Mentha insularis Requien & $\mathrm{H}$ & rept & no & arom & rare & ind \\
\hline 192 & Fabaceae & Mentha requienii Benth. & $\mathrm{H}$ & caesp & no & arom & rare & ind \\
\hline 193 & Euphorbiaceae & Mercurialis corsica Cosson & $\mathrm{Ch}$ & caesp & no & tox & v.r. & fav \\
\hline 194 & Lamiaceae & Micromeria cordata (Moris ex Bertol.) Moris & $\mathrm{H}$ & caesp & no & hab & rare & ind \\
\hline 195 & Lamiaceae & Micromeria filiformis (Aiton) Bentham & $\mathrm{T}$ & prost & yes & hab & rare & ind \\
\hline 196 & Brassicaceae & Morisia monantha (Viv.) Ascherson ex Barbey & $\mathrm{H}$ & ros & yes & no & rare & unfav \\
\hline 197 & Boraginaceae & Myosotis soleirolii Godr. in Gren. \& Godr. & $\mathrm{H}$ & scap & no & hab & rare & ind \\
\hline 198 & Asteraceae & Nananthea perpusilla (Loisel.) DC. & $\mathrm{H}$ & prost & no & hab & rare & fav \\
\hline 199 & Lamiaceae & Nepeta foliosa Moris & $\mathrm{Ch}$ & caesp & no & arom & rare & fav \\
\hline 200 & Scrophulariaceae & Odontites corsicus (Loisel.) G. Don & $\mathrm{T}$ & scap & yes & no & rare & fav \\
\hline 201 & Apiaceae & Oenanthe lisae Moris & $\mathrm{H}$ & caesp & yes & no & rare & unfav \\
\hline 202 & Orchidaceae & Ophrys $\times$ domus-maria Grasso & G & bulb & yes & no & rare & ind \\
\hline 203 & Orchidaceae & Ophrys $\times$ laconensis Scrugli \& Grasso nsubsp. laconensis & G & bulb & yes & no & rare & ind \\
\hline 204 & Orchidaceae & Ophrys $\times$ maremmae nsubsp. woodii Corrias & G & bulb & yes & no & rare & ind \\
\hline 205 & Orchidaceae & Ophrys annae Devillers-Tersch. & $\mathrm{G}$ & bulb & yes & no & rare & ind \\
\hline 206 & Orchidaceae & Ophrys chestermanii (Wood) Golz \& Reinhard & $\mathrm{G}$ & bulb & yes & no & rare & ind \\
\hline 207 & Orchidaceae & Ophrys scolopax subsp. conradiae (Melki \& Deschâtres) Baumann \& al. & G & bulb & yes & no & rare & ind \\
\hline 208 & Orchidaceae & Ophrys eleonorae Devillers-Tersch. \& Devillers & G & bulb & yes & no & rare & ind \\
\hline 209 & Orchidaceae & Ophrys morisii (Martelli) Soò in Keller \& al. & G & bulb & yes & no & rare & ind \\
\hline 210 & Orchidaceae & Ophrys holoserica subsp. chestermanii J.J. Wood & G & bulb & yes & no & rare & ind \\
\hline 211 & Orchidaceae & Ophrys morisii (Martelli) Soo in Keller \& al. & G & bulb & yes & no & rare & ind \\
\hline 212 & Orchidaceae & Ophrys panattensis Scrugli, Cogoni \& Pessei & G & bulb & yes & no & rare & ind \\
\hline 213 & Orchidaceae & Ophrys scolopax ssp. sardoa Baumann \& al. & G & bulb & yes & no & rare & ind \\
\hline 214 & Orchidaceae & Ophrys sphegodes Miller subsp. praecox Corrias & G & bulb & yes & no & rare & ind \\
\hline 215 & Orchidaceae & Orchis $\times$ penzigiana ssp. sardoa Scrugli \& Grasso & G & bulb & yes & no & rare & ind \\
\hline 216 & Orchidaceae & Orchis brancifortii Biv.-Bern. & $\mathrm{G}$ & bulb & yes & no & rare & ind \\
\hline 217 & Liliaceae & Orchis mascula subsp. ichnusae Corrias & G & bulb & yes & no & rare & ind \\
\hline 218 & Liliaceae & Ornithogalum corsicum Jordan & G & par & no & no & rare & fav \\
\hline 219 & Orobanchaceae & Orobanche denudata Moris & G & par & no & no & com. & ind \\
\hline 220 & Orobanchaceae & Orobanche australis Moris & G & par & no & no & v.r. & ind \\
\hline 221 & Orobanchaceae & Orobanche litorea Guss. & G & par & no & no & rare & ind \\
\hline 222 & Orobanchaceae & Orobanche rigens Loisel. & G & par & no & no & com. & fav \\
\hline 223 & Paeoniaceae & Paeonia morisii Cesca, Bernardo \& Passalacqua & G & scap & no & tox & com. & fav \\
\hline 224 & Amaryllidaceae & Pancratium illyricum $\mathrm{L}$. & G & bulb & yes & tox & com. & fav \\
\hline 225 & Poaceae & Phalaris rotgesii (Husnot) Baldini & $\mathrm{T}$ & caesp & yes & no & v.r. & unfav \\
\hline 226 & Poaceae & Phleum sardoum (Hackel) Hackel in Franchet & $\mathrm{T}$ & caesp & yes & hab & v.r. & ind \\
\hline 227 & Pinaceae & Pinus pinaster ssp. hamiltonii (Ten.) H. Del Villar & $\mathrm{P}$ & scap & yes & res & rare & ind \\
\hline 228 & Asteraceae & Plagius flosculosus (L.) Alavi \& Heywood & $\mathrm{H}$ & caesp & yes & no & rare & unfav \\
\hline 229 & Plantaginaceae & Plantago sarda C. Presl & $\mathrm{Ch}$ & caesp & yes & no & rare & fav \\
\hline 230 & Poaceae & Poa balbisi Parl. & $\mathrm{H}$ & scap & yes & no & spor. & fav \\
\hline 231 & Polygalaceae & Polygala sardoa Chodat & $\mathrm{H}$ & scap & yes & pulv & v.r. & ind \\
\hline 232 & Polygalaceae & Polygala sinisica Arrigoni & $\mathrm{Ch}$ & scap & yes & pulv & freq. & ind \\
\hline 233 & Polygonaceae & Polygonum scoparium Réq. ex Loisel. & $\mathrm{Ch}$ & caesp & yes & tox & rare & ind \\
\hline 234 & Rosaceae & Potentilla caulescens subsp. nebrodensis (Strobl ex Zimm.) Arrigoni & $\mathrm{H}$ & rept & yes & hab & rare & unfav \\
\hline 235 & Rosaceae & Potentilla crassinervia Viv. & $\mathrm{H}$ & rept & yes & no & rare & unfav \\
\hline 236 & Rosaceae & Potentilla corsica Soleir. ex Lehman & $\mathrm{Ch}$ & rept & yes & no & spor. & unfav \\
\hline
\end{tabular}


Table 1. continued.

\begin{tabular}{|c|c|c|c|c|c|c|c|c|}
\hline No. & Family & Taxon & 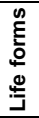 & 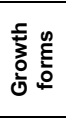 & $\begin{array}{l}\frac{7}{2} \\
\frac{7}{\pi} \\
\frac{0}{5} \\
\frac{0}{\pi} \\
0\end{array}$ & 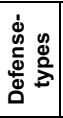 & 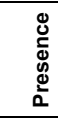 & 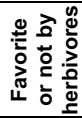 \\
\hline 236 & Rosaceae & Potentilla corsica Soleir. ex Lehman & Ch & rept & yes & no & spor. & unfav \\
\hline 237 & Fabaceae & Psoralea morisiana Pignatti \& Metlesics & $\mathrm{Ch}$ & caesp & no & hab & spor. & unfav \\
\hline 238 & Asteraceae & Ptilostemon casabonae (L.) Greuter & $\mathrm{H}$ & scap & no & spin & rare & ind \\
\hline 239 & Apiaceae & Ptychotis sardoa Pignatti \& Metlesics & $\mathrm{H}$ & scap & no & arom & rare & ind \\
\hline 240 & Ranunculaceae & Ranunculus cordiger Viviani subsp. cordiger & $\mathrm{H}$ & ros & yes & no & rare & ind \\
\hline 241 & Ranunculaceae & Ranunculus cordiger subsp. diffusus (Moris) Arrigoni & $\mathrm{H}$ & ros & yes & no & rare & ind \\
\hline 242 & Ranunculaceae & Ranunculus cymbalarifolius Balbis ex Moris & $\mathrm{H}$ & ros & yes & no & rare & ind \\
\hline 243 & Rhamnaceae & Rhamnus persicifolia Moris & $\mathrm{P}$ & caesp & yes & no & rare & unfav \\
\hline 244 & Saxifragaceae & Ribes sandalioticum (Arrigoni) Arrigoni & $\mathrm{P}$ & caesp & yes & no & punct. & unfav \\
\hline 245 & Saxifragaceae & Ribes sardoum Martelli & $\mathrm{P}$ & caesp & yes & no & v.r. & unfav \\
\hline 246 & Asteraceae & Hypochoeris robertia Fiori & $\mathrm{H}$ & ros & no & hab & com. & unfav \\
\hline 247 & Iridaceae & Romulea requienii Parl. & G & bulb & no & no & rare & fav \\
\hline 248 & Iridaceae & Romulea revelieri Jord. \& Fourr. & $\mathrm{G}$ & bulb & yes & no & rare & fav \\
\hline 249 & Rubiaceae & Rubia requienii Duby & $\mathrm{P}$ & lian & yes & spin & punct. & fav \\
\hline 250 & Rosaceae & Rubus arrigonii Camarda & $\mathrm{P}$ & suff & yes & spin & punct. & unfav \\
\hline 251 & Rosaceae & Rubus laconensis Camarda & $\mathrm{P}$ & suff & yes & spin & punct. & unfav \\
\hline 252 & Rosaceae & Rubus pignatti Camarda & $\mathrm{P}$ & suff & yes & spin & punct. & unfav \\
\hline 253 & Rosaceae & Rubus limbarae Camarda & $\mathrm{P}$ & suff & yes & spin & punct. & unfav \\
\hline 254 & Polygonaceae & Rumex suffocatus Moris ex Bertol. & $\mathrm{H}$ & ros & no & no & rare & fav \\
\hline 255 & Rutaceae & Ruta corsica DC. & $\mathrm{Ch}$ & caesp & no & tox & rare & fav \\
\hline 256 & Caryophyllaceae & Sagina pilifera (DC.) Fenzl & $\mathrm{H}$ & pulv & yes & no & rare & fav \\
\hline 257 & Salicaceae & Salix arrigonii Brullo & $\mathrm{P}$ & scap & yes & no & com. & ind \\
\hline 258 & Lamiaceae & Salvia desoleana Atzei \& Picci & $\mathrm{Ch}$ & caesp & no & arom & v.r. & ind \\
\hline 259 & Asteraceae & Santolina corsica Jord. \& Fourr. & $\mathrm{Ch}$ & caesp & no & arom & com. & fav \\
\hline 260 & Asteraceae & Santolina insularis (Gennari ex Fiori) Arrigoni & $\mathrm{Ch}$ & caesp & yes & arom & rare & fav \\
\hline 261 & Caryophyllaceae & Saponaria alsinoides (Viv.) Viv. & $\mathrm{H}$ & scand & yes & $h a b$ & rare & fav \\
\hline 262 & Saxifragaceae & Saxifraga cervicornis Viv. & $\mathrm{H}$ & caesp & no & hab & rare & ind \\
\hline 263 & Saxifragaceae & Saxifraga corsica (Ser. ex Duby) Gren. \& Godron & $\mathrm{H}$ & scap & yes & hab & rare & ind \\
\hline 264 & Hyacinthaceae & Scilla corsica Boullu & G & bulb & no & no & rare & fav \\
\hline 265 & Caryophyllaceae & Scleranthus burnatii Briquet & $\mathrm{H}$ & prost & yes & no & spor. & fav \\
\hline 266 & Asteraceae & Scorzonera callosa Moris & $\mathrm{H}$ & ros & no & no & spor. & ind \\
\hline 267 & Scrophulariaceae & Scrophularia morisii Valsecchi & $\mathrm{H}$ & scap & yes & tox & rare & fav \\
\hline 268 & Scrophulariaceae & Scrophularia oblongifolia Loisel. & $\mathrm{H}$ & rhiz & no & tox & rare & fav \\
\hline 269 & Scrophulariaceae & Scrophularia trifoliata L. & $\mathrm{H}$ & scap & no & tox & freq. & fav \\
\hline 270 & Crassulaceae & Sedum villosum subsp. glandulosum (Moris) P. Fourn. & $\mathrm{H}$ & caesp & no & hab & spor. & ind \\
\hline 271 & Orchidaceae & Serapias nurrica Corrias & $\mathrm{G}$ & bulb & yes & no & com. & ind \\
\hline 272 & Apiaceae & Seseli praecox (Gamisans) Gamisans & $\mathrm{Ch}$ & caesp & yes & hab & rare & ind \\
\hline 273 & Poaceae & Sesleria insularis subsp. barbaricina Arrigoni & $\mathrm{H}$ & caesp & yes & no & rare & fav \\
\hline 274 & Poaceae & Sesleria insularis subsp. morisiana Arrigoni & $\mathrm{H}$ & scap & yes & no & rare & fav \\
\hline 275 & Poaceae & Sesleria insularis Sommier subsp. insularis & $\mathrm{H}$ & scap & yes & no & rare & fav \\
\hline 276 & Caryophyllaceae & Silene beguinotii Vals. & $\mathrm{H}$ & rhiz & yes & no & rare & ind \\
\hline 277 & Caryophyllaceae & Silene corsica DC. & $\mathrm{H}$ & rhiz & yes & hab & rare & ind \\
\hline 278 & Caryophyllaceae & Silene martinolii Bocchieri \& Mulas & $\mathrm{T}$ & scap & yes & hab & rare & ind \\
\hline 279 & Caryophyllaceae & Silene morisiana Bég. \& Ravano & $\mathrm{H}$ & prost & yes & hab & rare & ind \\
\hline 280 & Caryophyllaceae & Silene nodulosa Viv. & $\mathrm{H}$ & prost & yes & hab & rare & ind \\
\hline 281 & Caryophyllaceae & Silene nummica Vals. & $\mathrm{H}$ & prost & yes & hab & rare & ind \\
\hline 282 & Caryophyllaceae & Silene sanctae-therasiae Jeanmonod & $\mathrm{Ch}$ & scap & yes & no & punct. & unfav \\
\hline 283 & Caryophyllaceae & Silene valsecchiae Bocchieri & $\mathrm{Ch}$ & scap & no & no & v.r. & ind \\
\hline 284 & Caryophyllaceae & Silene velutina Pourret ex Loisel. & $\mathrm{Ch}$ & caesp & no & hab & v.r. & ind \\
\hline 285 & Urticaceae & Soleirolia soleirolii (Réq.) Dandy & $\mathrm{H}$ & rept & no & hab & v.r. & ind \\
\hline 286 & Caryophyllaceae & Solenopsis minuta ssp. corsica Meikle & $\mathrm{H}$ & rept & no & hab & rare & ind \\
\hline 287 & Caryophyllaceae & Spergularia macrorhiza (Réq. ex Loisel.) Heynh. & $\mathrm{Ch}$ & rept & no & hab & com. & ind \\
\hline 288 & Lamiaceae & Stachys corsica Pers. & $\mathrm{H}$ & rept & yes & hab & com. & ind \\
\hline 289 & Lamiaceae & Stachys glutinosa L. & $\mathrm{Ch}$ & pulv & yes & arom & punct. & fav \\
\hline 290 & Lamiaceae & Stachys salisii Jord. \& Fourr. & $\mathrm{H}$ & rept & no & no & rare & ind \\
\hline 291 & Asteraceae & Tanacetum audiberti (Requien) DC. & $\mathrm{H}$ & caesp & no & arom & rare & fav \\
\hline 292 & Lamiaceae & Teucrium subspinosum Pourret ex Willd. & $\mathrm{Ch}$ & pulv & no & arom & rare & fav \\
\hline 293 & Santalaceae & Thesium italicum DC. & $\mathrm{T}$ & prost & yes & no & rare & ind \\
\hline 294 & Brassicaceae & Thlaspi brevistylum Jordan & $\mathrm{T}$ & scap & yes & no & com. & ind \\
\hline 295 & Lamiaceae & Thymus catharinae Camarda & $\mathrm{Ch}$ & pulv & yes & arom & com. & fav \\
\hline 296 & Poaceae & Trisetaria gracilis (Moris) Banfi \& Arrigoni & $\mathrm{H}$ & caesp & no & no & spor. & unfav \\
\hline 297 & Urticaceae & Urtica atrovirens Réq. ex Loisel. & $\mathrm{H}$ & scap & no & spin & spor. & fav \\
\hline 298 & Scrophulariaceae & Verbascum conocarpum Moris & $\mathrm{H}$ & scap & no & tox & rare & fav \\
\hline 299 & Scrophulariaceae & Verbascum plantagineum Moris & $\mathrm{T}$ & scap & yes & tox & rare & fav \\
\hline 300 & Scrophulariaceae & Veronica brevistyla Moris in Moris \& De Notaris & $\mathrm{T}$ & scap & no & no & spor. & fav \\
\hline 301 & Apocynaceae & Vinca sardoa (Stearn) Pignatti & $\mathrm{H}$ & scand & no & tox & rare & fav \\
\hline 302 & Violaceae & Viola limbarae (Merxm. \& Lippert) Arrigoni & $\mathrm{H}$ & scand & yes & hab & rare & fav \\
\hline
\end{tabular}




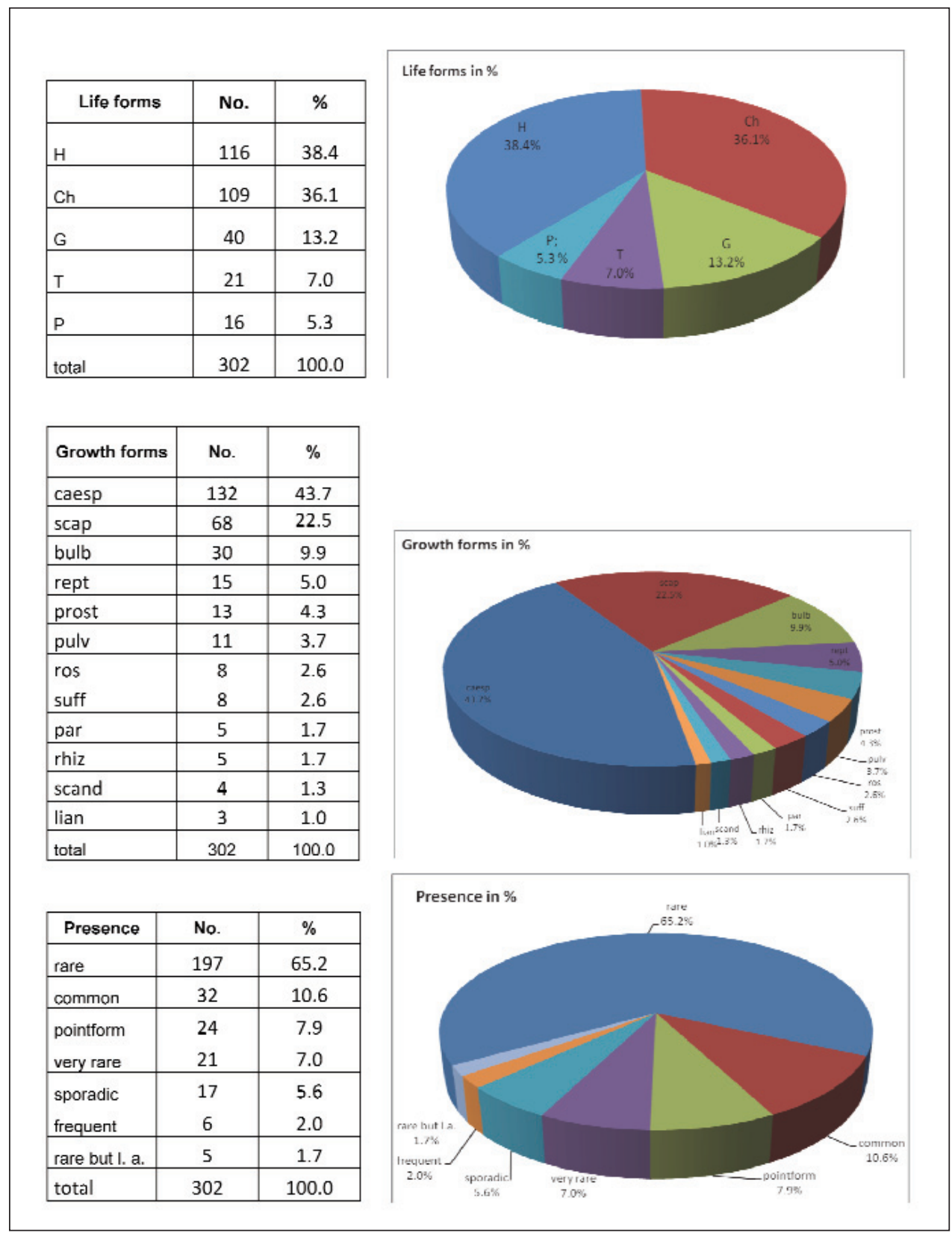

Fig. 1. Tables and related Pie charts expressed in $\%$ of the life forms, growth forms and their kind of presence. Abbreviations for Life forms: $\mathrm{H}=$ Hemicryptophyte, $\mathrm{Ch}=$ Chamaephyte, $\mathrm{G}=$ Geophyte, $\mathrm{T}=$ Therophyte, $\mathrm{P}=$ Phanerophyte.(rare but $1 . \mathrm{a} .=$ rare but locally abundant). Abbreviations for Growth forms: caesp $=$ cespitose, scap $=$ scapose, bulb $=$ bulbous, rept $=$ reptant, prost $=$ prostate, pulv $=$ pulvinate, ros $=$ rosulate, suff $=$ suffruticose, par $=$ parassite,, hiz $=$ rhizomatous, scand $=$ scan dent, lian = lianas. Abbreviations for Presence: rare but 1. a. = rare but locally abundant. 


\begin{tabular}{|c|c|c|}
\hline Pabularity & No. & $\%$ \\
\hline yes & 156 & 51.7 \\
\hline no & 146 & 48.3 \\
\hline total & 302 & 100.0 \\
\hline
\end{tabular}

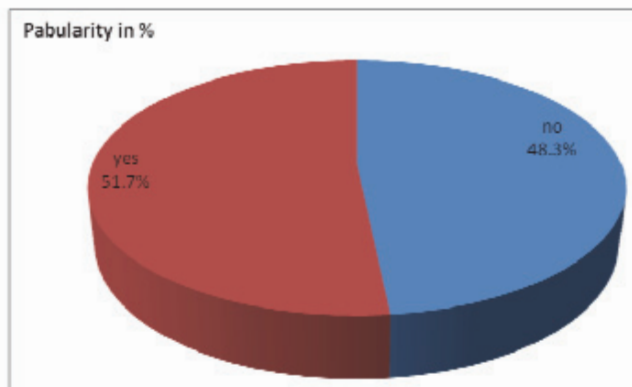

\begin{tabular}{|l|c|c|}
\hline $\begin{array}{c}\text { Defense } \\
\text { types }\end{array}$ & No. & $\%$ \\
\hline hab & 114 & 37.7 \\
no & 92 & 30.5 \\
\hline spin & 40 & 13.2 \\
\hline tox & 32 & 10.6 \\
arom & 21 & 7.0 \\
\hline pulv/bush & 2 & 0.7 \\
\hline res & 1 & 0.3 \\
\hline total & 302 & 100.0 \\
\hline
\end{tabular}

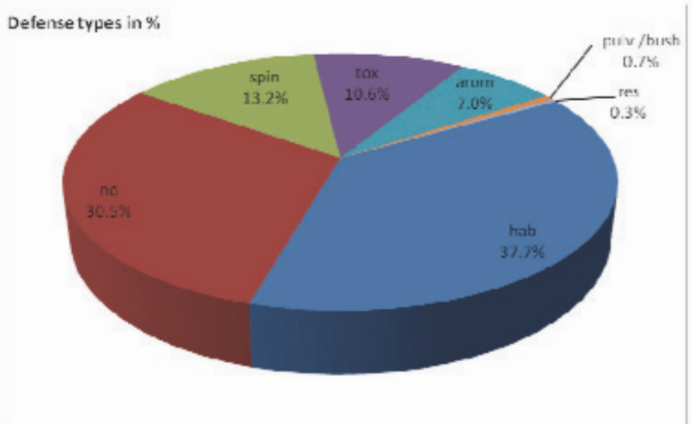

\begin{tabular}{|l|c|c|}
\hline $\begin{array}{c}\text { Favorite } \\
\text { or not } \\
\text { by } \\
\text { herbivores }\end{array}$ & No. & $\%$ \\
\hline ind & 137 & 45.4 \\
fav & 116 & 38.4 \\
\hline unfav & 49 & 16.2 \\
\hline total & 302 & 100.0 \\
\hline
\end{tabular}

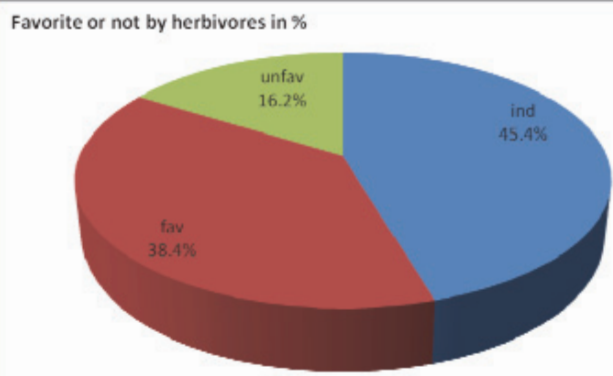

Fig. 2. Tables and relatives Pie charts expressed in \% of the pabularity, defense-types and favorite by grazing. Abbreviations for Defense types: hab $=$ habitus, spin $=$ spinescent, tox $=$ toxic, arom $=$ aromatic, pulv $/$ bush $=$ pulvinate/bush, res $=$ resinous. Abbreviations for Favorite or not by herbivores: ind $=$ indifferent, fav $=$ favorite, unfav $=$ unfavorite. 


\section{Aknowledgements}

We are grateful to Prof. Giuseppe Pulina (Departement of Agriculture of the University of Sassari) for his suggestions and input.

\section{References}

Arrigoni, P. V., Camarda, I., Corrias, B., Diana S., Nardi, E., Raffaelli M. \& Valsecchi, F. 1977-1991: Le piante endemiche della Sardegna: 1-202. - Boll. Soc. Sarda Sci. Nat. 16-28.

Aru, A., Baldaccini, P. \& Camarda, I. 1982: Ricerche pedologiche, floristiche e fenologiche sui pascoli del bacino del Rio s'Acqua Callenti (Villasalto, Sardegna sud-orientale). - Boll. Soc. Sarda Sci. Nat. 21: 199-283.

Camarda, I. 1977: Ricerche sulla vegetazione di alcuni pascoli montani del Marghine e del Supramonte di Orgosolo (Sardegna centrale). - Boll. Soc. Sarda Sci. Nat. 16: 215-250.

- 1984: L'influence humaine dans la chaîne calcaire du Monte Albo (Sardaigne centre-orientale). Coll. Phytosociol. (1984) 11: 511-522.

- 1989: Composizione floristica dei pascoli naturali del versante settentrionale del Marghine. - Pp. 53-83 in: Idda, L. (Ed.), Sistemi Agricoli Marginali - Sassari.

- 1989: Il censimento delle forme biologiche. - Pp. 19-26 in: Riunione scientifica su Problemi di rilevazione, interpretazione di flore locali. - Milano.

- 1990: Un approccio alla conoscenza dei pascoli naturali della Sardegna. - Pp. 30-59 in: Atti del Convegno Valorizzazione delle risorse produttive dei pascoli e salvaguardia dell'ambiente in Sardegna. - Cagliari.

Dell, A., Hopkins, A. J. M. \& Lamont, B. B. 1986: Resilience in mediterranean-type ecosystems. Dordrecht, Boston, Lancaster.

Di Castri, F.1981: Mediterranean type shrublands if the World. - Pp. 1-43 in: Di Castri, F., Goodall, D. W. \& Specht, R. L. (eds.). - Ecosystem of the World, 11. - Oxford.

Farris, E. \& Filigheddu, R. 2008: Effects of browsing in relation to vegetation cover on common yew (Taxus baccata L.) recruitment in Mediterranean environments. - Pl. Ecol. 199: 309-318.

-, Pisanu, S., Ceccherelli, G. \& Filigheddu, R. 2009: Effects of the management regime on the performance of the endangered Mediterranean Centaurea horrida Badarò (Asteraceae). - J. Nat. Conserv. 17: 15-24.

—, Filigheddu, R., Deiana, P., Farris, G. A. \& Garau, G. 2010: Short-term effects on sheep pastureland due to grazing abandonment in a Western Mediterranean island ecosystem: a multidisciplinary approach. - J. Nat. Conserv. 18: 258-267. doi: 10.1016/j.jnc.2009.11.003

Le Houerou, H. N. 1981: Impact of fire in the Mediterranean region. - Pp. 479-521 in: Di Castri, F., Goodall, D. W. \& Specht, R. L. (eds.). - Ecosystem of the World, 11. - Oxford.

Le Lannou, M. 1941: Pâtres et paysans de la Sardaigne. - Tours.

Naveh, Z. 1974: Effects of fire in the Mediterranean region. - Pp. 401-434 in: Kozlowski, T. \& Ahlgren, C. E. (eds.), Fire and Ecosystems. - New York.

Pisanu, S., Filigheddu, R. \& Farris, E. 2009: The conservation status of an endemic species of northern sardinia: Centaurea horrida Badarò (Asteraceae). - Pl. Biosyst. 143(2): 275-282. doi: 10.1080/11263500902722493

—, Farris, E., Filigheddu, R. \& Garcìa, M. B. 2012: Demographic effects of large, introduced herbivores on a long-lived endemic plant. - P1. Ecology 213: 1543-1553. doi: 10.1007/s11258-012-0110-9

Pulina, G. \& Biddau, G. 2015: Pascoli, pecore e politica: 70 anni di pastorizia in Sardegna. - Sassari. 
—, Rassu, S. P. G., Rossi, G. \& Brandano, P. 2011: La pastorizia sarda nell'ultimo secolo. - Pp. 11111131 in: Mattone, A. \& Simbula, P. F., La pastorizia mediterranea. Storia e diritto secoli XIXX. - Roma.

Tomaselli, R. 1976: La dégradation du maquis meditarréen: écologie, conservation et aménagement. - Notes Techn. MAB 2: 35-76.

Wilkens, B. 2012: Archeozoologia. Il Mediterraneo, la storia, la Sardegna. - Sassari.

Address of the authors :

Ignazio Camarda, Giuseppe Brundu, Luisa Carta, Gabriella Vacca \& Antonello Brunu, Departement of Agriculture of the University of Sassari, Via Piandanna 4, 07100Sassari, Italy. E-mail anbrunu@uniss.it 
